\title{
Effect of nicorandil on QT dispersion in patients with stable angina pectoris undergoing elective angioplasty: A triple-blind, randomized, placebo-controlled study
}

\author{
Homa Fal Suleimani ${ }^{1}$, Ali Eshraghi ${ }^{2}$, Mehdi Hasanzadeh Daloee ${ }^{3}$, Sara Hoseini ${ }^{4}$, Nima Nakhaee ${ }^{4}$
}

\author{
${ }^{1}$ M.D., Assistant Professor, Department of Cardiology, Atherosclerosis Prevention Research Center, Imam Reza \\ Hospital, Mashhad University of Medical Sciences, Mashhad, Iran \\ ${ }^{2}$ M.D., Assistant Professor, Department of Cardiology, Atherosclerosis Prevention Research Center, Ghaem \\ Hospital, Mashhad University of Medical Sciences, Mashhad, Iran \\ ${ }^{3}$ M.D., Professor, Department of Cardiology, Atherosclerosis Prevention Research Center, Ghaem Hospital, \\ Mashhad University of Medical Sciences, Mashhad, Iran \\ ${ }^{4}$ M.D., Resident of Cardiology, Department of Cardiology, Faculty of Medicine, Imam Reza Hospital, Mashhad \\ University of Medical Sciences, Mashhad, Iran
}

\section{Type of article: Original}

\begin{abstract}
Background: Nicorandil leads to the relaxation of fine vascular smooth muscle, and thus causes vasodilatation of major epicardial. Also, it has anti-arrhythmic and cardio-protective effects by improving reperfusion, and ultimately leads to a reduction in microvascular damage caused by percutaneous coronary intervention (PCI).

Objective: The aim of this study was to determine the effect of nicorandil on QT interval dispersion (QTd) in patients with stable angina pectoris during elective angioplasty.

Methods: This triple-blind and randomized clinical trial was performed on patients with stable angina pectoris, candidates for elective angiography referred to Imam Reza and Ghaem hospitals in Mashhad, Iran, between January and October 2016. The patients were randomly assigned to one of two groups receiving nicorandil (60 $\mathrm{mg}$ as $20 \mathrm{mg}$ before and $40 \mathrm{mg}$ after PCI) and placebo. All the patients underwent electrocardiography 12 hours before and 12 hours after PCI. The values of maximal corrected QT interval (QTc max) and QTd in these intervals, and the levels of changes in the QTd (QTd difference before angiography and after PCI) were compared between the two groups. Data were analyzed statistically using SPSS version 18 software via Chi-square and Independent-samples t-test.

Results: This study was performed on 90 patients ( 55 males and 35 females) with a mean age of $58.6 \pm 10.8$ years, on two groups of 45 people. The two groups were matched for age, body mass index, cardiovascular risk factors and baseline testing. The QTd before angiography had no statistically significant difference between the patients of both groups (control: $77.7 \pm 17.1$ vs. nicorandil: $80.7 \pm 14.2 \mathrm{~ms} ; \mathrm{p}=0.371$ ). The QTd after PCI in the nicorandil group was lower than the control group $(48.1 \pm 14.2$ vs. $59.2 \pm 15.6 \mathrm{~ms} ; \mathrm{p}=0.000)$. The decrease rate in QTd had a statistically significant difference between the two groups (control: $18.9 \pm 11.0$ vs. nicorandil: $33.5 \pm 9.5 \mathrm{~ms}$; $\mathrm{p}=0.000$ ).

Conclusions: The results of this study showed that oral administration of nicorandil around the PCI could further reduce QTd following PCI, compared to the control group.

Trial registration: The trial was registered at the Iranian Registry of Clinical Trials (http://www.irct.ir) with the Irct ID: IRCT2016120631159N1

Funding: The authors received no financial support for the research, authorship, and/or publication of this article. Keywords: Nicorandil, QT-dispersion, Percutaneous Coronary Intervention, Coronary Artery Disease
\end{abstract}

\section{Corresponding author:}

Dr. Nima Nakhaee, Department of Cardiology, Atherosclerosis Prevention Research Center, Faculty of Medicine, Imam Reza Hospital, Mashhad University of Medical Sciences, Mashhad, Iran.

Tel: +98.9155072379, Email: nakhaeenima@gmail.com

Received: January 11, 2017, Accepted: June 12, 2017, Published: August 2017

iThenticate screening: June 05, 2017, English editing: July 18, 2017, Quality control: August 10, 2017

This article has been reviewed / commented by three experts

(C) 2017 The Authors. This is an open access article under the terms of the Creative Commons Attribution-NonCommercialNoDerivs License, which permits use and distribution in any medium, provided the original work is properly cited, the use is non-commercial and no modifications or adaptations are made. 


\section{Introduction}

Myocardial tissue damage due to inadequate perfusion, is characterized by a relatively common complication following angioplasty $(1,2)$; a phenomenon that eventually leads to cardiac remodeling, heart failure and poor prognosis of patients $(2,3)$. So now, the ultimate goal in ischemic heart disease, especially acute myocardial infarction, in addition to vascular blood flow, is the establishment of myocardial perfusion (1-3). Nicorandil is a nicotinamide nitrate ester that, in addition to analgesic properties by opening ATP-sensitive potassium channel (or KATP channel), leads to the relaxation of fine vascular smooth muscle, and thus causes vasodilatation of major epicardial vessels and fine coronary artery $(4,5)$. It seems that the vasodilatation, also by improving the reperfusion, can prevent the development of phenomena such as slow flow and no flow in patients with percutaneous coronary intervention, (PCI) (6-8). Moreover, many studies have proven the cardio-protective and anti-arrhythmic effects of nicorandil following angioplasty in acute myocardial ischemia. However, studies on the efficacy of nicorandil following angioplasty in patients with stable angina pectoris are limited and imperfect, and its effectiveness has not been proven in this group of patients using electrophysiological criteria such as QT-dispersion (4, 9-12). QT interval dispersion (QTd) refers to the difference between minimum and maximum QTc values in a 12-lead electrocardiogram (ECG) that represents the electrical activity of ventricles from depolarization to repolarization $(13,14)$. Several studies have shown that higher QTd (greater dispersion in QT of different leads) is associated with the incidence of arrhythmia in various heart diseases, including coronary artery disease, long QT syndrome (LQTS) and heart failure (15-18). Its relationship has also been proven with heart ischemic events and sudden cardiac deaths (15-18). Several studies have found that the QTd value increases in myocardial infarction and its level is associated with heart muscle viability; successful treatment of ischemia ultimately leads to the reduction of QTd (15-18). Therefore, it can be used as a proper indicator in monitoring the myocardial microcirculation status (16-17). The aim of this study was to investigate the effect of oral administration of nicorandil before and after PCI compared with placebo in QTd changes after elective PCI in patients with stable angina pectoris.

\section{Material and Methods}

\subsection{Study design and setting}

This triple-blind and randomized clinical trial was performed on patients who were candidates for elective angiography, referred to Imam Reza and Ghaem hospitals in Mashhad, Iran, between January 2016 and October 2016.

\subsection{Participants}

Patients who were diagnosed with stable angina pectoris using history taking and patients who had definite indications for elective angiography were included into the study. Exclusion criteria included: 1) history of heart surgery, 2) having branch block with QRS $>120 \mathrm{~ms}$ or complete atrioventricular block in ECG, 3) atrial fibrillation, 4) renal failure or electrolyte abnormalities, 5) use of narcotics, 6) no need to perform angioplasty and 6) complications during angioplasty (acute stent thrombosis, coronary dissection, adjacent vessels lost and no reflow of the arteries).

\subsection{Sampling}

The sample size was determined to be 45 patients in each group according to the results of Ueda et al. (7), and twomean test formula for a quantitative trait in two independent societies. For sample size estimation, the values were considered as alpha $=5 \%$, beta $=80 \%, \mu_{1}=23.1, \mu_{2}=33.5, \mathrm{SD}_{1}=12.0, \mathrm{SD}_{2}=21.0, \mathrm{Z}_{1-\alpha / 2}=1.96$ and $\mathrm{Z}_{1-\beta}=0.84$. In this study, sampling was done by simple randomized method until completion of the sample size among patients entered into the study.

\subsection{Allocating and blinding}

The patients were randomly subjected into one of two intervention or control groups. Nurses in the angiography ward had appointed codes assigned to the patients and drug or placebo before starting the study; and the principal investigator of the project was therefore unaware of the medications available in numbered envelopes. The patients also were not aware of the type of interventions. For blinding the third level, the encoded groups were presented to a statistician for statistical analysis.

\subsection{Interventions}

The intervention group received a total of $60 \mathrm{mg}$ of nicorandil $20 \mathrm{mg}$ tablets (Merck, USA) orally in 3 intervals; first turn: 12 hours before angiography, second turn: 3 hours before angiography, and the last turn: 6 hours after angioplasty. The patients who merely underwent angiography with no need for angioplasty were not given the third 
dose and were excluded from the study. The control group was administered in the same way by isoshape, isocolor and isosmall placebo.

\subsection{Data collection}

At baseline, age, gender, body mass index, cardiovascular risk factors such as diabetes, high blood pressure, smoking, history of coronary artery diseases and dyslipidemia were recorded for the patients. Then, $10 \mathrm{cc}$ blood samples were taken from the patients to determine the levels of hemoglobin, creatinine, low-density lipoprotein (LDL) and high-density lipoprotein (LDL). Subsequently, all patients were given a two-dimensional echocardiogram, using a vivid 7 ultrasound machine (GE, USA) performed by a cardiologist. The left ventricular ejection fraction was recorded for the patients before angiography. Also, before the administration of the first dose of the drug or placebo, the patients underwent electrocardiography using a Fukuda ME C110 device (Japan). Next, the patients underwent angiography, diagnosed by Artis zee (Siemens, Germany) by a cardiac interventionist, and the pattern of coronary artery involvement was found. Based on the diagnosis of the interventionist, the patients underwent angioplasty. Then, the types of coronary intervention, the number of vessels under stenting and stent length were recorded.

\subsection{QTd calculation}

The QT interval was calculated from the beginning of the QRS complex to the end of the $\mathrm{T}$ wave on the basis of millisecond (ms). The corrected QT (cQT) was calculated based on heart rate using the MDCalc C) 2016 software. The QTd was obtained based on the difference between minimum and maximum QTc values in the 12-lead ECG in ms. The QTd changes manually, based on the difference between the QTd values before and after PCI was determined by a fourth-year resident of cardiology.

\subsection{Outcomes}

The main outcome of this study was to determine the QTd changes after elective PCI in patients receiving nicorandil in comparison with placebo. The second outcome was to compare QTd changes after elective PCI in patients receiving nicorandil in comparison with placebo in different subgroups of gender, types of intervention, number implanted stents and stent size.

\subsection{Ethical considerations}

This study was approved by the ethics committee of Mashhad University of Medical Sciences (code of ethics: IR.MUMS.fm.REC.1394.152). The content of the project was explained to all patients before entering the study, and their information was used in the project with patient satisfaction. All patients signed the consent form. Privacy and dignity of patients were also considered in the project. Information on patients was inserted into the statistical analysis software as encoded, and was published as overall results (registration code of IRCT: IRCT2016120631159N1).

\subsection{Statistical analysis}

Information required for the project was extracted based on checklists. Data were analyzed statistically using SPSS version 18 software (PASW Statistics for Windows, Chicago: SPSS Inc.). Analysis of Independent-samples t-test was used to measure quantitative data and chi-square analysis for qualitative data. The confidence interval was considered $95 \%$.

\section{Results}

\subsection{Baseline characteristics}

This study was performed on 90 patients (55 males and 35 females) with the mean age of $58.2 \pm 10.8$ years in two groups of 45 people (Figure 1). There was no statistically significant difference between the two groups in terms of age, gender, cardiovascular risk factors, body mass index, left ventricular ejection fraction and baseline biochemical tests (Table 1).

\subsection{QTd findings}

There was no statistically significant difference between the two groups in terms of max cQT and QTd before angiography, but the results showed that the values of cQT max and QTd after angiography were significantly lower in the nicorandil group compared with the control group (Table 2). The reduction level of QTd after angioplasty was also significantly higher in the nicorandil group (Table 2). The mean reduction level of QTd after angioplasty was $26.2 \pm 12.6 \mathrm{~ms}$ in all the studied patients. 


\subsection{QTd changes in subgroups}

The results of this study showed that the mean difference of QTd before angiography relative to after angioplasty had statistically significant difference among all sub-groups of male and female, the number of stents implanted and the presence or absence of a history of diabetes between the nicorandil and control groups under study (Table 3). There was no statistically significant difference among all sub-groups of the type of intervention only in the subgroup of more than two-vessel stenting, and among all sub-groups of stent sizes, the cases more than $45 \mathrm{~mm}$ between the nicorandil and control groups (Table 3).

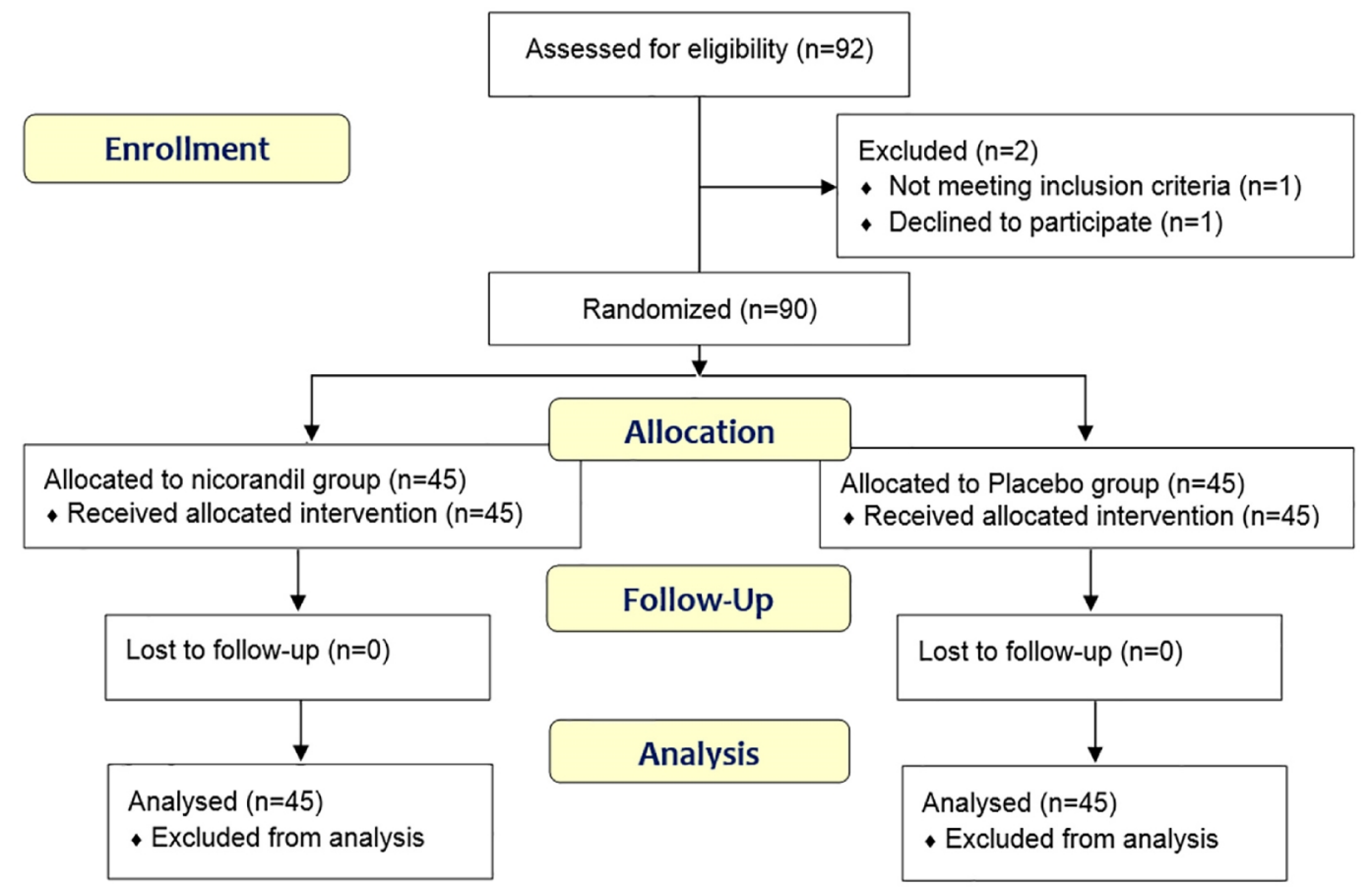

Figure 1. Follow-up diagram of patients (According to consort statement)

Table 1. Comparison of underlying variables between the two nicorandil and control groups under study

\begin{tabular}{|c|c|c|c|c|}
\hline Variables, unit & Total & $\begin{array}{l}\text { Control group } \\
(\mathrm{n}=45)\end{array}$ & $\begin{array}{l}\text { Nicorandil group } \\
(\mathrm{n}=45)\end{array}$ & p-value \\
\hline Age, (years); mean \pm SD & $58.6 \pm 10.8$ & $59 . \pm 10.0$ & $58.1 \pm 11.6$ & 0.672 \\
\hline BMI, $\mathrm{kg} / \mathrm{m}^{2} ;$ mean $\pm \mathrm{SD}$ & $26.1 \pm 3.9$ & $25.7 \pm 3.2$ & $26.6 \pm 4.3$ & 0.296 \\
\hline $\mathrm{Hb}, \mathrm{g} / \mathrm{dL} ;$ mean $\pm \mathrm{SD}$ & $13.2 \pm 1.2$ & $13.0 \pm 1.4$ & $13.3 \pm 0.9$ & 0.183 \\
\hline Creatinine, mg/dL; mean \pm SD & $1.3 \pm 1.2$ & $1.3 \pm 0.5$ & $1.2 \pm 0.3$ & 0.501 \\
\hline LDL (Low-density lipoprotein), mg/dL; mean \pm SD & $107.2 \pm 36.8$ & $105.9 \pm 34.5$ & $108.0 \pm 36.0$ & 0.843 \\
\hline HDL (High-density lipoprotein), mg/dL; mean \pm SD & $41.6 \pm 9.0$ & $42.0 \pm 10.7$ & $41.3 \pm 8.0$ & 0.817 \\
\hline Number of vessels under stenting; mean \pm SD & $1.3 \pm 0.5$ & $1.2 \pm 0.5$ & $1.4 \pm 0.6$ & 0.079 \\
\hline Stent sizes, $\mathrm{mm} ;$ mean $\pm \mathrm{SD}$ & $33.7 \pm 21.2$ & $32.4 \pm 20.6$ & $35.0 \pm 21.9$ & 0.564 \\
\hline Ventricular ejection fraction, percentage; mean $\pm \mathrm{SD}$ & $47.0 \pm 12.8$ & $47.6 \pm 12.0$ & $46.3 \pm 13.7$ & 0.643 \\
\hline \multirow{2}{*}{ Gender, $\mathrm{n}(\%)$} & Male & 23 & 32 & \multirow[t]{2}{*}{0.052} \\
\hline & Female & 22 & 13 & \\
\hline \multirow[t]{2}{*}{ Smoking, n (\%) } & Yes & 16 & 12 & \multirow[t]{2}{*}{0.362} \\
\hline & No & 29 & 33 & \\
\hline \multirow[t]{2}{*}{ History of heart disease, $\mathrm{n}(\%)$} & Yes & 16 & 11 & \multirow[t]{2}{*}{0.250} \\
\hline & No & 29 & 34 & \\
\hline \multirow[t]{2}{*}{ Dyslipidemia, n (\%) } & Yes & 18 & 12 & \multirow[t]{2}{*}{0.180} \\
\hline & No & 27 & 33 & \\
\hline \multirow[t]{2}{*}{ High blood pressure, $\mathrm{n}(\%)$} & Yes & 21 & 27 & \multirow[t]{2}{*}{0.205} \\
\hline & No & 24 & 18 & \\
\hline \multirow[t]{2}{*}{ Diabetes, n (\%) } & Yes & 20 & 19 & \multirow[t]{2}{*}{0.832} \\
\hline & No & 25 & 26 & \\
\hline
\end{tabular}


http://www.ephysician.ir

Table 2. Comparison of corrected QT and QTd before angiography, after PCI, and QTd difference after PCI relative to before angiography, between nicorandil and control groups under study

\begin{tabular}{|l|l|l|l|}
\hline Variables, unit & $\begin{array}{l}\text { Control group } \\
(\mathrm{n}=45)\end{array}$ & $\begin{array}{l}\text { Nicorandil group } \\
(\mathrm{n}=45)\end{array}$ & $\begin{array}{l}\mathrm{p}- \\
\text { value }\end{array}$ \\
\hline cQT max before angiography, ms & $406.9 \pm 45.5$ & $405.7 \pm 34.1$ & 0.892 \\
\hline cQT max after PCI*, ms & $430.0 \pm 34.2$ & $390.6 \pm 30.3$ & 0.001 \\
\hline QTd before angiography, ms & $77.7 \pm 17.1$ & $80.7 \pm 14.2$ & 0.371 \\
\hline QTd after PCI, ms & $59.2 \pm 15.6$ & $48.1 \pm 14.2$ & 0.001 \\
\hline $\begin{array}{l}\text { QTd difference after PCI relative to before } \\
\text { angiography, ms }\end{array}$ & $18.9 \pm 11.0$ & $33.5 \pm 9.5$ & 0.001 \\
\hline
\end{tabular}

* PCI: Percutaneous coronary intervention

Table 3. Comparison of QT-dispersion difference before angiography relative to after PCI, between nicorandil and control groups

\begin{tabular}{|c|c|c|c|c|}
\hline \multirow[t]{2}{*}{ Subgroups } & \multirow[t]{2}{*}{ Variables } & \multicolumn{2}{|c|}{$\begin{array}{l}\text { Difference of primary and final QTd, } \\
\text { Mean } \pm \text { SD }\end{array}$} & \multirow[t]{2}{*}{ p-value } \\
\hline & & Control group $(n=45)$ & $\begin{array}{l}\text { Nicorandil group } \\
(\mathrm{n}=45)\end{array}$ & \\
\hline \multirow[t]{2}{*}{ Gender } & Male & $20.5 \pm 11.8$ & $34.8 \pm 9.1$ & 0.001 \\
\hline & Female & $17.3 \pm 10.3$ & $30.4 \pm 10.1$ & 0.001 \\
\hline \multirow[t]{2}{*}{ Diabetes } & Yes & $20.2 \pm 11.5$ & $37.3 \pm 9.5$ & 0.001 \\
\hline & No & $18.0 \pm 10.8$ & $32.7 \pm 9.5$ & 0.001 \\
\hline \multirow[t]{4}{*}{ Type of intervention } & LAD stent & $22.0 \pm 9.9$ & $34.4 \pm 6.6$ & 0.001 \\
\hline & LCX stent & $15.5 \pm 11.7$ & $31.4 \pm 4.9$ & 0.007 \\
\hline & RCA stent & $13.5 \pm 5.7$ & $30.6 \pm 6.1$ & 0.000 \\
\hline & $\begin{array}{l}\text { More than two-vessel } \\
\text { stent }\end{array}$ & $25.3 \pm 12.0$ & $35.3 \pm 12.9$ & 0.061 \\
\hline \multirow{2}{*}{$\begin{array}{l}\text { Number of implanted } \\
\text { stents }\end{array}$} & 1 & $18.3 \pm 9.5$ & $32.8 \pm 6.4$ & 0.001 \\
\hline & 2 & $23.6 \pm 12.1$ & $36.4 \pm 10.8$ & 0.004 \\
\hline \multirow{2}{*}{ Stent sizes $(\mathrm{mm})$} & $\leq 45$ & $17.6 \pm 9.2$ & $34.7 \pm 8.4$ & 0.004 \\
\hline & $>45$ & $28.1 \pm 11.7$ & $31.4 \pm 12.8$ & 0.571 \\
\hline
\end{tabular}

\section{Discussion}

The results of this study revealed that although the QTd value after PCI declined in both groups, the reduction level of QTd, after prescribed oral Nicorandil drug, was higher than that of the control group. According to recent studies, further reduction of QTd is associated with a better blood supply to myocardial tissue. Therefore, this study provides further evidence of the effectiveness of Nicorandil to improve tissue perfusion after PCI and to reduce damages caused by angioplasty. Another important point is that, the QTd value after PCI was reported respectively about 48 $\mathrm{ms}$ and $60 \mathrm{~ms}$ in the nicorandil and control groups; the difference was also statistically significant, indicating further evidence of the effectiveness of the nicorandil drug. A study by Rotterdam in a four-year follow-up, showed that patients with QTd less than $39 \mathrm{~ms}$ had a lower risk of total mortality, cardiac death and sudden death compared with those with values over $60 \mathrm{~ms}$ (19). Moreover, in another study, the cardiovascular mortality was significantly higher in patients with QT dispersion over $58 \mathrm{~ms}$ compared to those with below $58 \mathrm{~ms}$ (20). Very few studies have examined the effect of Nicorandil based on QTd changes, especially in patients with stable angina pectoris. Therefore, our results can be an important aid in determining the position of Nicorandil in the PCI of the patients with stable angina pectoris. In our study, further reduction in the QTd value was shown by increasing the number of vessels under stenting (the control and nicorandil groups); and if the stent is placed on the LCX and LAD, the QTd value will be faced with the greatest rate of decline. This finding appears to be due to increased levels of myocardial tissue perfusion, although no study has been done on the relationship among the QTd changes with the type of vessel and status of the stenting to discuss on its generalization. It should be noted that the high level of decrease in the QTd is clinically significant in patients undergoing LAD and LCX stents compared to the patients who were under the RCA stent, while the type of vessel under stent in our study had no influence statistically on the effectiveness of nicorandil. The interesting thing is that when stenting is performed on more than two vessels, we are faced with the greatest reduction of QTd in the control group. This rate of decline was so close to the reduction in the nicorandil group, which made no significant difference for the changes in the QTd value in these patients 
between the two groups. However, no hypothesis can be raised easily on the cause of the phenomenon; but it seems that one can infer that in cases where additional myocardial tissue perfusion is made by two stents, it is probable that improvement in myocardial perfusion has occurred even in the absence of nicorandil. It should be mentioned that the use of nicorandil in this group of patients has led to a further reduction in QTd (about $10 \mathrm{~ms}$ ) than in the control group. In addition to our study, other studies have shown the efficacy of nicorandil on the reduction of QT dispersion during PCI; for example, the study of Ueda et al (8). In this study, QT dispersion within 48 hours after PCI, was shorter in the nicorandil group compared with the control group (QT dispersion, respectively, $16.1 \pm 23.2$ ms vs. $24 \pm 33.4 \mathrm{~ms}, \mathrm{p}<0.05$ ). However, the Ueda study was conducted on patients with acute myocardial ischemia and this study differed to ours in this respect, because our study was performed on patients with stable angina pectoris. In addition, in one study conducted on patients with stable angina pectoris (5), it was shown that $15 \mathrm{mg}$ of oral Nicorandil led to a significant reduction in the increase of QTd after the first PCI for the patients on the LAD, though QT dispersion after reperfusion was similar for both groups. Perhaps the most important difference between our study and the study of Kato (5) can be attributed to the differences in dose, as well as the differences in the target group studied, since Kato examined only patients with LAD involvement. In our study, for the first time, in addition to the LAD, QTd changes were evaluated in other vascular subgroups. Nicorandil reduces the increase in QT dispersion during PCI with different mechanisms. The most important mechanism of action of the direct effect of nicorandil drugs is opening up a KATP channel in the shortest time (6), nitric oxide release and protective effects on the heart by reducing the entrance of excessive amounts of $\mathrm{Ca}^{2+}$ into the muscle tissue cells (21). Due to the activities of nitrate, nicorandil leads to stimulation of guanylate cyclase and increase in cyclic GMP formation (cGMP), which ultimately reduces smooth muscle sensitivity to calcium through downstream signaling cascade, and leads to dilation of blood vessels and increases coronary blood flow. In parallel, with the opening of K-ATP channels by nicorandil without preventing a rise in intracellular sodium level and reduced intracellular ATP and phosphocreatine during ischemia, mitochondrial respiration is established and improvement in the concentration of ATP in the tissues after ischemia is accelerated (22). In addition, it is proposed that antioxidant activities of nicorandil lead to a protective effect on coronary artery endothelial cells (1). The results of a systematic review of Multi et al. (10) on more than 1,600 patients showed that the nicorandil drug improves coronary artery reperfusion, stops irregular heartbeats caused by reperfusion and improves left ventricular function in patients with AMI who had experienced primary PCI. It should be noted that our study has been carried out on patients with stable angina pectoris and perhaps some of the mechanisms are not fully consistent with the conditions of the disease. It is also known that nicorandil drug use prior to reperfusion time improves coronary artery thrombolysis in myocardial infarction (TIMI) flow, prevents irregular heartbeat and improves left ventricular function (10). In addition, it is reported that protein kinase $\mathrm{C}$ causes the opening of the KATP channel and then induces preconditioning effect (23).

One of the strengths of our study, over other studies, was the comparison of QTd changes in various subgroups. Previous studies have reported a correlation between gender and the differences in QT intervals (24). Therefore, it was important that we examine this hypothesis in this study. Kassotis et al. (25) reported that men had a higher QT dispersion, but women had longer QT interval and less QT dispersion. But Ueda et al. (7) found no relationship between gender and QT dispersion. Our study also showed that QTd changes occurred significantly in both genders and the rate of change in both genders was almost equal. In addition, nicorandil in both genders was more effective, and resulted in a further reduction in QTd value compared to the control group. Another factor considered in this study was the role of presence or absence of a risk factor of diabetes, and its impact on the effectiveness of intervention. It was shown that diabetes has no association with the influence of nicorandil, a finding that has not been mentioned in other studies. In addition, our study demonstrated that in patients with stent size less than $45 \mathrm{~mm}$ compared to stent size more than $45 \mathrm{~mm}$, nicorandil was more effective. In the stent size more than $45 \mathrm{~mm}$, in spite of further myocardial damage caused by angioplasty, it was expected that nicorandil might be more beneficial, while this was not the case based on statistical analysis. The possible reason for this phenomenon may be attributable to low sample sizes or inability of impact of nicorandil in these cases with greater damages. Nevertheless, this finding needs further studies with larger sample size. However, other studies have used indicators other than QTd to determine the efficacy of nicorandil, which the differences in methodologies make that we do not review them in this study. Among these studies, we can refer to a study of Kim et al. (26), who took advantage of troponin and creatine kinase-MB. The results showed that the nicorandil drug has myocardial protective effect during percutaneous coronary intervention in patients with unstable angina. In another study, Yang et al. (11) using troponin, showed that administration of oral nicorandil $(10 \mathrm{and} 20 \mathrm{mg})$ two hours before PCI reduces the incidence of cardiac damages during PCI and heart attack associated with PCI. The most recent study has also revealed that the use of nicorandil reduces ischemic attacks in patients with stable angina pectoris (12). 


\section{Limitations and suggestions}

There are several limitations in this study. First, it was carried out on a minimum possible sample size. To generalize the results of this research, further studies should be conducted with larger sample size. Second, follow-up duration was short. It is better to examine the effectiveness and possible side effects of nicorandil at longer intervals. Third, the QT intervals were measured manually, though there is no evidence of a more precise measurement of QT interval with a computer compared to manual measurement (24). For generalization of the efficacy of nicorandil in myocardial perfusion improvement in the patients undergoing PCI, it is recommended that more studies should be done in other countries, because the majority of studies that have been done so far are restricted mostly to Japan and more recently Iran.

\section{Conclusions}

Oral administrations of $60 \mathrm{mg}$ nicorandil around PCI in patients with stable angina pectoris can cause further reduction of QTd compared with placebo following PCI. Therefore, according to the available evidence, it appears that nicorandil could improve myocardial perfusion and reduce ventricular recovery time. However, there is still need for further research with larger samples, especially in non-Asian countries, particularly in patients with stable angina pectoris, to further generalized effectiveness of oral nicorandil on the results of PCI.

\section{Trial registration:}

The trial was registered at the Iranian Registry of Clinical Trials (http://www.irct.ir) with the Irct ID: IRCT2016120631159N1.

\section{Funding:}

The authors received no financial support for the research, authorship, and/or publication of this article.

\section{Conflict of Interest:}

There is no conflict of interest to be declared.

\section{Authors' contributions:}

All authors contributed to this project and article equally. All authors read and approved the final manuscript.

\section{References:}

1) Gang Y, Guo X, Crook R, Hnatkova K, Camm A, Malik M. Computerised measurements of QT dispersion in healthy subjects. Heart. 1998; 80(5): 459-66. doi: 10.1136/hrt.80.5.459. PMID: 9930044, PMCID: PMC1728844.

2) Partovi N, Falsoleiman H. Nicorandil in patients with acute coronary syndrome and stable angina undergoing percutaneous coronary intervention: literature review. Reviews in Clinical Medicine. 2014; 2(1): 42-4.

3) Gholoobi A, Sajjadi SM, Shabestari MM, Eshraghi A, Shamloo AS. The Impact of Remote Ischemic PreConditioning on Contrast-Induced Nephropathy in Patients Undergoing Coronary Angiography and Angioplasty: A Double-Blind Randomized Clinical Trial. Electron physician. 2015; 7(8): 1557-65. doi: 10.19082/1557. PMID: 26816582, PMCID: PMC4725407.

4) Hirohata A, Yamamoto K, Hirose E, Kobayashi Y, Takafuji H, Sano F, et al. Nicorandil prevents microvascular dysfunction resulting from PCI in patients with stable angina pectoris: a randomised study. EuroIntervention. 2014; 9(9): 1050-6. doi: 10.4244/EIJV9I9A178. PMID: 24457276.

5) Kato T, Kamiyama T, Maruyama Y, Tanaka S, Yoshimoto N. Nicorandil, a potent cardioprotective agent, reduces QT dispersion during coronary angioplasty. Am Heart J. 2001; 141(6): 940-3. doi: 10.1067/mhj.2001.114369. PMID: 11376307.

6) Kobayashi Y, Miyata A, Tanno K, Kikushima S, Baba T, Katagiri T. Effects of nicorandil, a potassium channel opener, on idiopathic ventricular tachycardia. J Am Coll Cardiol. 1998; 32(5): 1377-83. doi: 10.1016/S0735-1097(98)00372-6. PMID: 9809951.

7) Ueda H, Hayashi T, Tsumura K, Yoshimaru K, Nakayama Y, Yoshikawa J. Intravenous nicorandil can reduce QT dispersion and prevent bradyarrhythmia during percutaneous transluminal coronary angioplasty of the right coronary artery. J Cardiovasc Pharmacol Ther. 2004; 9(3): 179-84. doi: 10.1177/107424840400900305. PMID: 15378138. 
8) Ueda H, Nakayama Y, Tsumura K, Yoshimaru K, Hayashi T, Yoshikawa J. Intravenous nicorandil can reduce the occurrence of ventricular fibrillation and QT dispersion in patients with successful coronary angioplasty in acute myocardial infarction. Can J Cardiol. 2004; 20(6): 625-9. PMID: 15152293.

9) Suematsu Y, Murasato Y, Miura Si, Horiuchi M, Yamamoto T, Takata K, et al. Safety and feasibility of high-dose administration of nicorandil before reperfusion therapy in acute myocardial infarction. Cardiovascular intervention and therapeutics. 2013; 28(4): 352-61. doi: 10.1007/s12928-013-0182-z. PMID: 23625617.

10) $\mathrm{Wu} \mathrm{M}$, Huang $\mathrm{Z}$, Xie H, Zhou Z. Nicorandil in patients with acute myocardial infarction undergoing primary percutaneous coronary intervention: a systematic review and meta-analysis. PloS one. 2013; 8(10): e78231. doi: 10.1371/journal.pone.0078231. PMID: 24167609, PMCID: PMC3805586.

11) Yang J, Zhang J, Cui W, Liu F, Xie R, Yang X, et al. Cardioprotective effects of single oral dose of nicorandil before selective percutaneous coronary intervention. Anatol J Cardiol. 2015; 15(2): 125-31. doi: 10.5152/akd.2014.5207. PMID: 25252296.

12) Jiang J, Li Y, Zhou Y, Li X, Li H, Tang B, et al. Oral nicorandil reduces ischemic attacks in patients with stable angina: A prospective, multicenter, open-label, randomized, controlled study. Int J Cardiol. 2016; 224: 183-7. doi: 10.1016/j.ijcard.2016.08.305. PMID: 27657471.

13) Day CP, McComb JM, Campbell R. QT dispersion: an indication of arrhythmia risk in patients with long QT intervals. Br Heart J. 1990; 63(6): 342-4. doi: 10.1136/hrt.63.6.342. PMID: 2375895, PMCID: PMC1024518.

14) Malik M, Batchvarov VN. Measurement, interpretation and clinical potential of QT dispersion. J Am Coll Cardiol. 2000; 36(6): 1749-66. doi: 10.1016/S0735-1097(00)00962-1. PMID: 11092641.

15) Shah CP, Thakur RK, Reisdorff EJ, Lane E, Aufderheide TP, Hayes OW. QT dispersion may be a useful adjunct for detection of myocardial infarction in the chest pain center. Am Heart J. 1998; 136(3): 496-8. doi: 10.1016/S0002-8703(98)70226-1. PMID: 9736143.

16) Ashikaga T, Nishizaki M, Arita M, Yamawake N, Suzuki M, Hashimoto Y, et al. Effect of dipyridamole on QT dispersion in vasospastic angina pectoris. The American journal of cardiology. 1999; 84(7): 807-10. doi: 10.1016/S0002-9149(99)00441-5.

17) Stolt A, Karila T, Viitasalo M, Mäntysaari M, Kujala UM, Karjalainen J. QT interval and QT dispersion in endurance athletes and in power athletes using large doses of anabolic steroids. Am J Cardiol. 1999; 84(3): 364-6. doi: 10.1016/S0002-9149(99)00299-4. PMID: 10496458.

18) Brendorp B, Elming H, Jun L, Køber L, Torp - Pedersen C. Effect of dofetilide on QT dispersion and the prognostic implications of changes in QT dispersion for patients with congestive heart failure. European journal of heart failure. 2002; 4(2): 201-6. doi: 10.1016/S1388-9842(01)00235-5.

19) Macfarlane P, editor. QT dispersion-Lack of discriminating power. Circulation. USA, Philadelphia: Lippincott Williams \& Wilkins; 1998.

20) Okin PM, Devereux RB, Howard BV, Fabsitz RR, Lee ET, Welty TK. Assessment of QT interval and QT dispersion for prediction of all-cause and cardiovascular mortality in American Indians the Strong Heart Study. Circulation. 2000; 101(1): 61-6. doi: 10.1161/01.CIR.101.1.61. PMID: 10618305.

21) Higham P, Furniss S, Campbell R. QT dispersion and components of the QT interval in ischaemia and infarction. Br Heart J. 1995; 73(1): 32-6. doi: 10.1136/hrt.73.1.32. PMID: 7888257, PMCID: PMC483752.

22) Fukuda H, Luo CS, Gu X, Guo LL, Digerness SB, Li J, et al. The effect of K ATP channel activation on myocardial cationic and energetic status during ischemia and reperfusion: role in cardioprotection. J Mol Cell Cardiol. 2001; 33(3): 545-60. doi: 10.1006/jmcc.2000.1328. PMID: 11181022.

23) Light PE, Sabir AA, Allen BG, Walsh MP, French RJ. Protein Kinase C-Induced Changes in the Stoichiometry of ATP Binding Activate Cardiac ATP-Sensitive K+ Channels A Possible Mechanistic Link to Ischemic Preconditioning. Circ Res. 1996; 79(3): 399-406. doi: 10.1161/01.RES.79.3.399. PMID: 8781473

24) Rautaharju P, Zhou S, Wong S, Calhoun H, Berenson G, Prineas R, et al. Sex differences in the evolution of the electrocardiographic QT interval with age. Can J Cardiol. 1992; 8(7): 690-5. PMID: 1422988.

25) Kassotis J, Costeas C, Bedi AK, Tolat A, Reiffel J. Effects of aging and gender on QT dispersion in an overtly healthy population. Pacing Clin Electrophysiol. 2000; 23(7): 1121-6. doi: 10.1111/j.15408159.2000.tb00912.x. PMID: 10914368.

26) Kim JH, Jeong MH, Yun KH, Kim KH, Kang DK, Hong SN, et al. Myocardial protective effects of nicorandil during percutaneous coronary intervention in patients with unstable angina. Circ J. 2005; 69(3): 306-10. doi: 10.1253/circj.69.306. PMID: 15731536. 